\title{
Resultados clínicos de Cirugía Refractiva (CR) PPK y LASIK, en Cuenca Ecuador, enero 2015-julio 2017
}

\section{Clinical results of Refractive Surgery (CR) PPK and LASIK, in Cuenca Ecuador, January 2015-July 2017}

\author{
Marisa Arcos $^{1,2} *$, Cesar Andres Matute Orellana $^{1}$ y Dayra Tatiana Siranaula Arias ${ }^{1}$ \\ ${ }^{1}$ Universidad Católica de Cuenca \\ ${ }^{2}$ Universidad del Salvador \\ *marisaarcos@hotmail.com
}

DOI: https://doi.org/10.26871/killkana_salud.v2i2.99

\begin{abstract}
Resumen
Contexto: Los errores refractarios constituyen el $50 \%$ del deterioro de la visión y el $33 \%$ de ceguera evitable a nivel mundial. Afectando la vida de los pacientes que los padecen, el objetivo de la CR es modificar la curvatura de la córnea, cambiando su poder de convergencia, mejorando la calidad visual. Ecuador no tiene estudios que revelen la efectividad de CR por las técnicas PRK y LASIK. Objetivo: Determinar los resultados de las técnicas CR por láser de femtosegundo por PRK y LASIK. Materiales y Métodos: Estudio descriptivo, retrospectivo, de corte transversal. Se revisaron historias clínicas de 87 pacientes (164 ojos), que fueron intervenidos por las técnicas PRK y LASIK en el periodo enero 2005 a junio 2017 en la Clínica Exiláser (Cuenca Ecuador). El procesamiento de datos fue realizado con SPSS 22. Resultados: La mayor frecuencia de defectos refractivos fue entre los 18 a 25 años de edad (34,5\%), el sexo femenino presentó el $60,9 \%$ de errores refractarios. La CR más usada fue LASIK en un 65,2\%. La ametropía más común, fue el astigmatismo miópico en un 54,3\%. Luego de CR hubo una mejoría en los valores de la curvatura corneal, además de la agudeza visual alcanzando valores de 20/20 y 20/25 en un 96,3\%. Conclusiones: CR es una opción terapéutica que provee de resultados positivos, produciendo un cambio psicosocial en las personas y dando una mejor calidad de vida. Logrando agudezas visuales de 20/20.
\end{abstract}

Palabras clave: Cirugía refractiva, Agudeza visual, PRK, LASIK, Femtosegundo.

\begin{abstract}
Context: Refractive errors constitute 50\% of vision impairment and 33\% of avoidable blindness worldwide, affecting the lives of the patients who suffer from these conditions. The objective of $C R$ is to modify the corneal curvature, changing its converging power, and improving visual quality. Ecuador does not have revealing studies on the effectiveness of CR by PRK and LASIK techniques. Objective: To determine the results of the femtosecond laser CR techniques through PRK and LASIK. Materials and Methods: Descriptive, retrospective, and cross-sectional study. The medical records of 87 patients (164 eyes), who were intervened using the PRK and LASIK techniques during the period January 2005 to June 2017, in the Exiláser Clinic in Cuenca, Ecuador; were analyzed. The data processing was performed using SPSS 22. Results: The highest frequency of refractive errors corresponded to those aged between 18 and 25 years (34.5\%), where the female sex presented $60.9 \%$ of refractory errors. The most used CR technique was LASIK (65.2\%). The most common ametropia was myopic astigmatism with 54.3\%. After applying CR, there was an improvement in the values of the corneal curvature, in addition to the visual acuity, reaching values of 20/20 and 20/25 in 96.3\%. Conclusions: CR is a therapeutic option that provides positive results, generating a psychosocial change in people, providing them with a better quality of life, and achieving visual acuity of 20/20.
\end{abstract}

Key words: Refractive surgery, Visual acuity, PRK, LASIK, Femtosecond.

\section{Introducción}

La visión ocurre cuando los rayos de la luz se desvían, es decir que son refractados, al pasar a través de la córnea y el cristalino. ${ }^{1,2}$ Esta luz es enfocada luego sobre la retina, quien transforma la luz en impulsos eléctricos que se envían al cerebro a través del nervio óptico, el cerebro interpreta estos mensajes convirtiéndolos en las imágenes que se ven. $^{3,4}$

El estado de refracción depende de cuatro factores principales: el poder corneal, el poder del cristalino, la profundidad de la cámara anterior y la longitud axial. ${ }^{5}$ 
La emetropía es una condición fisiológica, en donde el ojo en un estado de reposo y sin acomodación, enfoca las imágenes de los objetos y las proyecta desde la parte anterior del ojo exactamente sobre la retina y el individuo es capaz de percibir nítidamente esas imágenes, cuando la luz proviene de una distancia de 6 metros aproximadamente, entra al sistema óptico visual y se focaliza en la retina. ${ }^{6}$

Sin embargo, cuando los rayos de luz se enfocan fuera de la retina, se produce un defecto en la percepción visual denominado ametropía o defecto refractivo. ${ }^{7}$

El $50 \%$ del deterioro evitable de la visión y el $30 \%$ de la ceguera evitable a nivel mundial son debidas a errores de refracción. Alrededor de 150 millones de personas tienen baja agudeza visual por errores de refracción que no han sido corregidos, siendo su mayor prevalencia en países de bajos recursos económicos ${ }^{8}$

Estos defectos, se clasifican de acuerdo sus características de focalización y condiciones anatómicas del globo ocular donde se pueden clasificar en Miopía, Hipermetropía y Astigmatismo. ${ }^{9}$

\subsection{Miopía}

Es un trastorno refractivo muy común, ocurre cuando la imagen que ingresa por el ojo miope se enfoca delante de la retina por lo tanto produce una visión borrosa o desenfocada para la visión de lejos y el paciente presenta una buena visión de cerca. Se produce cuando la longitud axial del ojo es demasiado larga, siendo esta, la distancia desde la superficie corneal posterior a la retina ${ }^{10}$

La miopía se clasifica en 4 tipos $^{11}$

- Miopía baja:<-3.00 dioptrías

- Miopía moderada: -3.00 a -6.00 dioptrías

- Miopía severa: -6.00 -9.00 dioptrías

- Miopía extrema: >-9.00 dioptrías

\subsection{Astigmatismo}

El astigmatismo se refiere a la condición refractiva en la que una superficie corneal deformada hace que los rayos de luz que entran en el ojo a lo largo de diferentes planos se enfoquen de manera desigual; esto ocurre cuando existe diferente poder dióptrico en los meridianos, la córnea se curva más en una dirección que la otra. El paciente refiere que presenta una visión borrosa en todas las distancias de visualización. ${ }^{9}$

Los grados de astigmatismo: ${ }^{11}$

- Astigmatismo leve: $<1.00$ dioptría

- Astigmatismo moderado: 1.00 a 2.00 dioptrías

- Astigmatismo severo: 2.00 a 3.00 dioptrías

- Astigmatismo extremo: $>3.00$ dioptrías

\subsection{Hipermetropía}

Es un trastorno refractivo que ocurre cuando las imágenes que entra al ojo se enfocan por detrás de la retina, de tal manera que se produce una visión borrosa de cerca y una buena visión de lejos. ${ }^{12}$
Esta se corrige con un dispositivo refractivo que proporciona una superficie de refracción más convexa con el final de aumentar el poder de enfoque deficiente en el sistema óptico del paciente. ${ }^{13}$

- Hipermetropía baja: <+2.00 dioptrías

- Hipermetropía moderada: +2.00 a +4.00 dioptrías

- Hipermetropía severa: +4.00 a +6.00 dioptrías

- Hipermetropía extrema: >+6.00 dioptrías

\subsubsection{Tratamiento}

El tratamiento va de acuerdo a las necesidades y los síntomas del paciente, el mismo que va dirigido a mejorar la agudeza visual.

Dentro del tratamiento incluyen las lentes correctivas, como lentes de montura y lentes de contacto o cirugía refractiva. $^{14}$

\subsection{Cirugía Refractiva}

En los últimos 20 años se ha producido un avance en las técnicas oftalmológicas, y en los tratamientos oculares debidos a mejores equipos, mejores técnicas, sin embargo todo procedimiento refractivo depende de la respuesta de la córnea ante la agresión quirúrgica, dentro de la cual la que más resalta es la técnica de LASIK ha sido considerada como segura, eficaz y predecible ${ }^{15}$

Criterios a evaluar en el paciente que solicite cirugía refractiva:

Esta va a depender de una evaluación preoperatoria tanto ocular como sistémica.

- Historia clínica completa donde incluyan antecentes personales y familiares, uso de medicamentos, historia social.

- Se requiere solicitar exámenes de laboratorio, como Biometría Hemática completa, tiempos de protrombina, tromboplastina, plaquetas y glucosa.

- El examen oftalmológico preoperatorio con la determinación de agudeza visual corregida y no corregida

- Refracción bajo cicloplegia

- Espesor corneal por ultrasonido paquimetrica

- Queratometría manual, topografía corneal por videoqueratografia examen de lámpara de hendidura. ${ }^{16}$

Criterios de selección para paciente que se someterá a cirugía por medio de LASIK o PRK

- $\quad$ Error de refracción estable con variación de $\pm 0,50 \mathrm{D}$ en los 12 meses anteriores. Con miopía o hipermetropía con o sin astigmatismo, o astigmatismo mixto.

- Miopía de <-12.00 dioptrías con la técnica de LASIK y con Miopía <-6 dioptrías para la técnica de PRK.

- Astigmatismo de 5 dioptrías

- Hipermetropía de +6.00 dioptrías

- Ausencia de contraindicaciones

- Se recomienda que los pacientes candidatos a LASIK tengan al menos 2 años de visión estable (menos de 0,5 $\mathrm{D}$ de variación) con el uso de lentes de montura o lentes de contacto antes de someterse a cirugía. 


\subsubsection{Contraindicaciones}

Entre las contraindicaciones incluyen:

- Queratocono o una anormal topografía corneal.

- Enfermedades vasculares de colágeno, autoinmune o inmunodeficiencia.

- Mujeres embarazadas o en lactancia.

- El tratamiento con isotretinoína o clorhidrato de amiodarona

El ojo seco preoperatorio se ha tomado como un factor de riesgo, debido que más del $95 \%$ de los pacientes presentan este síntoma después del primer mes de cirugía y los síntomas suelen mejorar entre 6 a 12 meses postoperatorio, donde este se vuelve resistente al tratamiento. ${ }^{17}$

La alergia ocular es una contraindicación absoluta para la cirugía refractiva de láser, el glaucoma no controlado es una contraindicación absoluta hasta que no se tenga una presión intraocular normal antes de la cirugía ya que el uso de la técnica de LASIK tiene la desventaja de causar una elevación a corto pazo de la presión intraocular con el microqueratomo. ${ }^{16}$

La catarata en desarrollo con frecuencia causa cambios refractivos que prestan un transitorio e inexacto efecto del tratamiento $^{18}$

\subsection{Técnicas Quirúrgicas}

Existen varias técnicas quirúrgicas pero las más utilizadas son la de "photorefractive keratectomy" o PRK por sus siglas y la de"Laser Assited in situ keratomileusis" más conocida como LASIK. ${ }^{19}$

\subsubsection{PRK}

Es una técnica de laser superficial que consiste en la descamación superficial mediante el uso de eximer laser, la cual realizar una debridacion de la porción central del epitelio corneal con la finalidad de exponer el estroma de la córnea. Es un procedimiento en el que el epitelio es eliminado mecánicamente mediante raspado y posterior a esto, se aplica laser sobre la capa de Bowman para producir ablación, el estroma anterior y dejar así el área desnuda para que reepitelice, se coloca finalmente un lente de contacto blando para controlar el crecimiento epitelial, hasta que la córnea este reepitelizada, el epitelio de la zona central de la córnea es removido dejando un defecto epitelial aproximadamente de 6 a $8 \mathrm{~mm}$ de diámetro que cicatriza en un plazo de 3 a 5 días debido a la migración del epitelio circundante.(8-20)

El paciente puede experimentar un dolor más o menos intenso, el mismo que puede ser tratado con antiinflamatorios no esteroideos de forma tópica y con analgésicos orales. $^{20}$

\subsubsection{LASIK}

En esta técnica se realiza un corte lamelar de la córnea, con un tallado de la córnea mediante el uso de láser. En la actualidad, dicho corte, genera un colgajo corneal o el denominado flap corneal de 110 micras de espesor, que permanece unido a la córnea, por un extremo se levanta para poder dejar expuesto el estroma corneal que recibirá la energía producida por el equipo de láser. Esta técnica ofrece varias ventajas sobre las demás debido a que produce una recuperación más rápida en comparación al PRK. ${ }^{21}$

El procedimiento se realiza en dos instantes: el primero es la colocación de un anestésico tópico, luego se realiza dos marcas de tipo asimétricos sobre la córnea con un material de genciana; estas marcas permiten reconocer las marcas de flap al momento de concluir la cirugía. Luego se procede a la creación del colgajo corneal, flap o lenticulo estromal, utilizando un microqueratomo o el que actualmente se usa el denominado femtosegundo. Este emite un haz infrarrojo de una onda aproximada de $1053 \mathrm{~nm}$ que son pequeños pulsos de energía que impactan a una profundidad preseleccionada en el estroma corneal provocando una fotodisrupcion del tejido, en donde la presión y la temperatura elevada hacen que se produzcan numerosas micro cavidades en el estroma, generando de esta manera el colgajo. ${ }^{19}$

La respuesta de presentación cicatrizar a nivel corneal depende de varios factores como es la ablación de la superficie o de la lesión simultanea del epitelio y del estroma, el grado de lesión estromal, la utilización de fármacos que modulan la respuesta inflamatoria y cicatrizal y de factores individuales que determinan las diferencias entre dos sujetos ante una misma agresión ${ }^{22}$

La técnica de LASIK mantiene las estructuras anatómicas prácticamente intactas salvo en los márgenes donde se realiza el flap lo que conlleva a un menos estímulo para la respuesta cicatricial corneal. ${ }^{23}$

\section{Objetivo}

Determinar los resultados de las técnicas quirúrgicas de la cirugía refractiva por láser de femtosegundo por PRK y LASIK en la Clínica Exiláser, durante el período de enero 2015 a junio 2017 en la ciudad de Cuenca.

\section{Materiales y métodos}

Estudio descriptivo, retrospectivo, de corte transversal, en el que se les incluyó a 87 pacientes (164 ojos), de los que se recolectaron los datos de la revisión de historias clínicas de pacientes intervenidos en el periodo comprendido desde enero 2015 a junio 2017 de la Clínica Exiláser.

Se seleccionaron aquellos pacientes que fueron intervenidos quirúrgicamente por las técnicas PRK y LASIK, en base al formulario de datos sustentado en las variables de análisis. 


\section{Resultados}

Tabla 1. Distribución de pacientes según la técnica de Cirugía refractiva. Clínica Exiláser. Cuenca. Enero 2015 - Junio 2017

\begin{tabular}{|l|c|c|}
\hline Cirugía Refractiva & $\begin{array}{c}\text { Frecuencia } \\
\left(\mathbf{N}^{\circ}\right)\end{array}$ & $\begin{array}{c}\text { Porcentaje } \\
(\%)\end{array}$ \\
\hline LASIK & 107 & 65,2 \\
PRK & 57 & 34,8 \\
Total (ojos) & 164 & 100,0 \\
\hline
\end{tabular}

Fuente: Historias Clínicas

Elaborado: Dayra Tatiana Siranaula Arias

Interpretación: Como se muestra en la tabla 1, la mayoría de pacientes del estudio se han realizado la cirugía refractiva por la técnica de LASIK en un $65,2 \%(\mathrm{n}=107)$ y por la técnica de PRK en un $34,8 \%$ ( $\mathrm{n}=57)$, constituyendo un total de 164 ojos operados con cirugía refractiva por las técnicas de PRK y LASIK.

Tabla 2. Distribución de pacientes según el defecto refractivo. Clínica Exiláser. Cuenca. Enero 2015 - Junio 2017

\begin{tabular}{|l|c|c|}
\hline Defecto refractivo & $\begin{array}{c}\text { Frecuencia } \\
\left(\mathrm{N}^{\circ}\right)\end{array}$ & $\begin{array}{c}\text { Porcentaje } \\
(\%)\end{array}$ \\
\hline Astigmatismo hipermetrópico & 7 & 4,3 \\
Astigmatismo miópico & 89 & 54,3 \\
Astigmatismo mixto & 0 & 0,0 \\
Hipermetropia & 4 & 2,4 \\
Miopía & 64 & 39,0 \\
Total (ojos) & 164 & 100,0 \\
\hline
\end{tabular}

Fuente: Historias Clínicas

Elaborado: Dayra Tatiana Siranaula Arias

Interpretación: Como se muestra en la tabla 2, la mayoría de pacientes del estudio que fueron intervenidos por cirugía refractiva por la técnica de PRK y LASIK presentaron Astigmatismo miópico en un 54,3\% $(n=89)$, seguido de Miopía con un 39,0\% ( $n=64)$, y los defectos refractivos menos frecuentes que acudieron fueron Astigmatismo hipermetrópico con un 4,3\% (n=7), e Hipermetropía que fue el menos frecuente con un $2,4 \%(n=4)$.
Tabla 3. Distribución de pacientes según la edad. Clínica Exiláser. Cuenca. Enero 2015 - junio 2017

\begin{tabular}{|c|c|c|}
\hline Edad & $\begin{array}{c}\text { Frecuencia } \\
\left(\mathrm{N}^{\circ}\right)\end{array}$ & $\begin{array}{c}\text { Porcentaje } \\
(\%)\end{array}$ \\
\hline $18-25$ & 30 & 34,5 \\
$26-30$ & 8 & 9,2 \\
$31-35$ & 6 & 6,9 \\
$36-40$ & 9 & 10,3 \\
$41-45$ & 8 & 9,2 \\
46 o más & 26 & 29,9 \\
Total (pacientes) & 87 & 100,0 \\
\hline
\end{tabular}

Fuente: Historias Clínicas

Elaborado: Dayra Tatiana Siranaula Arias

Interpretación: Como se muestra en la tabla 3, la mayoría de pacientes del estudio que se han realizado la cirugía refractiva fueron entre las edades de 18 a 25 años con un $34,5 \%(n=30)$, seguido con un $29,9 \%(n=26)$ en mayores de 46 años. Y la minoría de pacientes con un 6,9\% $(n=6)$ entre las edades de 31 a 35 años. Observándose que las mayores frecuencias se encuentran en los dos extremos de la escala establecida.

Tabla 4. Distribución de pacientes según el sexo. Clínica Exiláser. Cuenca Enero 2015 - Junio 2017

\begin{tabular}{|l|c|c|}
\hline Sexo & $\begin{array}{c}\text { Frecuencia } \\
\left(\mathrm{N}^{\circ}\right)\end{array}$ & $\begin{array}{c}\text { Porcentaje } \\
(\%)\end{array}$ \\
\hline Femenino & 53 & 60,9 \\
Masculino & 34 & 39,1 \\
Total (pacientes) & 87 & 100,0 \\
\hline
\end{tabular}

Fuente: Historias Clínicas

Elaborado: Dayra Tatiana Siranaula Arias

Interpretación: Como se muestra en la tabla 4, el sexo femenino se realiza Cirugía refractiva con más frecuencia que el sexo masculino, observándose un 60,9\% $(\mathrm{n}=53)$ del sexo femenino y un $39,1 \%(n=34)$ del sexo masculino. 
Tabla 5. Distribución de pacientes según las Complicaciones postoperatorias. Clínica Exiláser. Cuenca. Enero 2015 - Junio 2017

\begin{tabular}{|l|r|r|}
\hline $\begin{array}{l}\text { Complicaciones } \\
\text { postoperatorias }\end{array}$ & $\begin{array}{r}\text { Frecuencia } \\
\left(\mathrm{N}^{\circ}\right)\end{array}$ & $\begin{array}{r}\text { Porcentaje } \\
(\%)\end{array}$ \\
\hline $\begin{array}{l}\text { Regresión del defecto } \\
\text { refractivo }\end{array}$ & 0 & 0,0 \\
Ectasia corneal & 2 & 0,0 \\
Haze corneal & 0 & 1,2 \\
Epitelización & & 0,0 \\
prolongada & 0 & 0,0 \\
Úlcera corneal & 0 & 0,0 \\
Infección & 161 & 0,6 \\
Ojo seco & 164 & 100,0 \\
No complicaciones & & 98,2 \\
Total (ojos) & & \\
& & \\
\hline
\end{tabular}

Fuente: Historias Clínicas

Elaborado: Dayra Tatiana Siranaula Arias

Interpretación: Como se muestra en la tabla 5, la mayoría de pacientes no presentaron ninguna complicación postoperatoria en un $98,2 \%(n=161)$; tan solo el $1,2 \%$ $(n=2)$ presentaron ojo seco y el $1,2 \%(n=2)$ haze corneal.

Tabla 6. Distribución de pacientes según la Agudeza visual sin corrección prequirúrgica y la Agudeza visual sin corrección postquirúrgica. Clínica Exiláser. Cuenca. Enero 2015 - Junio 2017

\begin{tabular}{|l|c|c|c|c|}
\hline Agudeza visual & \multicolumn{2}{|c|}{$\begin{array}{c}\text { Agudeza visual sin } \\
\text { corrección prequirúrgica }\end{array}$} & \multicolumn{2}{c|}{$\begin{array}{c}\text { Agudeza visual sin } \\
\text { corrección postquirúrgica }\end{array}$} \\
\cline { 2 - 5 } & $\begin{array}{c}\text { Frecuencia } \\
\left(\mathrm{N}^{\circ}\right)\end{array}$ & $\begin{array}{c}\text { Porcentaje } \\
(\%)\end{array}$ & $\begin{array}{c}\text { Frecuencia } \\
\left(\mathrm{N}^{\circ}\right)\end{array}$ & $\begin{array}{c}\text { Porcentaje } \\
(\%)\end{array}$ \\
\hline $20 / 20-20 / 25$ & 12 & 7,3 & 158 & 96,3 \\
\hline $20 / 50-20 / 80$ & 11 & 6,7 & 5 & 3,0 \\
\hline $20 / 100-20 / 160$ & 52 & 31,7 & 1 & 0,6 \\
\hline$\geq 20 / 200$ & 101 & 61,6 & 0 & 0,0 \\
\hline
\end{tabular}

$\mathrm{p}=0.042 \mathrm{n}=164$ (ojos)

Fuente: Historias Clínicas

Elaborado: Dayra Tatiana Siranaula Arias

Interpretación: Como se muestra en la tabla 6, existe una mejoría en la agudeza visual, presentando la mayoría de pacientes valores entre 20/20 - 20/25 en el 96,3\% ( $n=158)$, seguido de una minoría con agudeza visual entre 20/50 - 20/80 en un $3,0 \%(n=5)$, a diferencia de los valores prequirúrgicos sin corrección en que la mayoría mantenía una agudeza visual mayor o igual a 20/200 en un $61,6 \%$ $(\mathrm{n}=101)$. Demostrándose un valor significativo $\mathrm{p}=0,042$.

Tabla 7. Distribución de pacientes según la satisfacción postquirúrgica. Clínica Exiláser. Cuenca. Enero 2015 - Junio 2017

\begin{tabular}{|l|c|c|}
\hline \multicolumn{1}{|c|}{ Satisfacción } & $\begin{array}{c}\text { Frecuencia } \\
\left(\mathrm{N}^{\circ}\right)\end{array}$ & $\begin{array}{c}\text { Porcentaje } \\
(\%)\end{array}$ \\
\hline $\begin{array}{l}\text { Insuficientemente } \\
\text { satisfecho }\end{array}$ & 0 & 0,0 \\
$\begin{array}{l}\text { Medianamente } \\
\text { satisfecho }\end{array}$ & 15 & 17,2 \\
$\begin{array}{l}\text { Totalmente } \\
\text { satisfecho }\end{array}$ & 72 & 82,8 \\
Total (pacientes) & 87 & 100,0 \\
\hline
\end{tabular}

Fuente: Historias Clínicas

Elaborado: Dayra Tatiana Siranaula Arias

Interpretación: Como se muestra en la tabla 7, la mayoría de pacientes quedaron totalmente satisfechos posterior a la cirugía refractiva con un $82,8 \%(n=72)$ y tan solo con un $17,2 \%(\mathrm{n}=15)$ medianamente satisfecho.

\section{Discusión}

Dentro de las estimaciones globales indican que alrededor de 312 millones de personas son miopes en el año 2015, cifra que puede ascender a 324 millones para el año 2025 y a 4,758 millones para el año $2050 .^{24}$ Según el reporte de los países que conforman la Red Epidemiológica Iberoamericana para la Salud Visual y Ocular, indican que en el periodo 2009-2010, la mayor prevalencia es en Ecuador con un $25,2 \%$, y la menor prevalencia se reportó en Argentina con un $1.2 \% .^{24}$

Del total de casos de discapacidad visual, el $80 \%$ se pueden evitar o curar. Dentro de la distribución mundial de las principales causas de discapacidad visual son: errores de refracción no corregidos (miopía, hipermetropía o astigmatismo) en un $43 \%$; cataratas no operadas en un $33 \%$; glaucoma en un $2 \%$.

En el año 2013, la Asamblea Mundial de la Salud aprobó el Plan de acción para la prevención de la ceguera y la discapacidad visual, en el periodo 2014-2019, a modo de hoja de ruta para los Estados Miembros, la Secretaría de la OMS y los asociados internacionales, con el objetivo de lograr una reducción mensurable de la discapacidad visual evitable de un $25 \%$ de aquí al año 2019. ${ }^{22}$ La mayor parte en un $43 \%$ de las principales causas de discapacidad visual en el mundo corresponden a errores de refracción no corregidos como la miopía, hipermetropía o el astigmatismo. ${ }^{25}$

Múltiples investigaciones acerca de la cirugía refractiva láser mediante las técnicas de PRK y LASIK, han indicado 
resultados refractivos y visuales favorables $(26,27)$. En el presente estudio, el objetivo no fue comparar las técnicas, pero se evaluó la mayor frecuencia de las técnicas aplicadas, en donde se encontró que la mayoría de pacientes del estudio se han realizado la cirugía refractiva por la técnica de LASIK. Ambas técnicas presentan resultados similares, difiriendo entre ellas, las condiciones y criterios que presente el paciente para que el cirujano elija entre una y otra técnica, siendo en la actualidad la más utilizada por la técnica LASIK.

En estudios se demuestra que la técnica LASIK es la predominante en cirugía refractiva, ya que permite tratar con buenos resultados las ametropías bajas y moderadas. Y mediante la técnica de PRK, también permite tratar ese rango de ametropías, pero con limitaciones en los grados de ametropías altas, es ideal especialmente en casos de córneas delgadas, u otra condición que justifique evitar la construcción de un flap corneal $(21,24)$. Aunque según Perdomo F, la técnica de LASIK ha llegado a ser la cirugía electiva más común, con más de 35 millones de procedimientos ejecutados alrededor del mundo para el año 2010. Se puede evidenciar que este procedimiento ha logrado corregir el $96 \%$ de los errores refractivos con mínimas incomodidades para el paciente, con un tiempo de recuperación de pocas horas, y con excelentes resultados. ${ }^{25}$

En el estudio, se dividieron las edades por grupos de 5 años sin importar el sexo, condición social, o religión, para la división de los adultos jóvenes se consideró las edades desde los 18 hasta los 25 años de edad. De acuerdo a los resultados obtenidos se puede describir que la edad con más predisposición a los defectos refractivos que fueron intervenidos por cirugía refractiva por las técnicas de PRK y LASIK en la Clínica Exiláser con un universo de 87 pacientes fueron jóvenes. En el estudio de Ortega L, con un universo de 257 pacientes (504 ojos), cuya muestra quedó conformada por 31 pacientes (59 ojos), de los cuales el $45,2 \%$ presentaron edades entre 30 y 39 años, con una edad media fue de $32,5 \pm 7,7$ años (rango entre 21 y 48 años), ${ }^{26}$ cuyas edades no concuerda con nuestro estudio, en donde la minoría fue entre las edades de 31 a 35 años, con un $6,9 \%$. Mientras que en el estudio de Mian S, la edad de mayor frecuencia hace similitud con nuestro estudio, con resultados obtenidos en Estados Unidos y la Europa occidental, donde afectan aproximadamente un tercio de las personas de edad $\geq 40$ años. $^{13}$

Debido a que según varios estudios que concluyen, en que la edad de mayor frecuencia de defectos refractivos es mayor a los 40 años; la Academia Americana de Oftalmología recomienda exámenes oftalmológicos para adultos sin signos o factores de riesgo, a partir de los 40 años. Las personas $\sin$ factores de riesgo entre edades de 40 a 54 años deben ser examinados cada dos o cuatro años, entre edades de 55 a 63 años cada uno a tres años, y los mayores de 65 años cada uno a dos años. Además, cualquier paciente con antecedentes oculares, médicos o familiares se deben realizar exámenes periódicos, debido al riesgo individual (20-26,29)

En la distribución de los pacientes según los defectos refractivos sobresale el Astigmatismo miópico y miopía, los defectos refractivos menos frecuentes que acudieron fueron Astigmatismo hipermetrópico e Hipermetropía. Concuerda con el estudio de Perdomo, agosto 2012 a setiembre 2013, en donde se sometieron a cirugía con láser excimer, 954 personas (1701 ojos), de los cuales, para este estudio, 218 personas fueron miopes, que en su mayoría fueron mujeres $(61 \%) .{ }^{25}$ Como se muestra la mayor frecuencia es el sexo femenino que fueron intervenidos por cirugía refractiva que el sexo masculino. Concuerda con el estudio realizado por Ortega L, en donde más de la mitad de los pacientes fueron del sexo femenino en un $54,8 \%{ }^{25}$

Como antes se expone, la prevalencia de la miopía se ha reportado tan alta como se muestra del 70-90\% en algunos países asiáticos, del 30-40\% en Europa y América, y $10-20 \%$ en África. En Ecuador se efectuó el estudio en 1,283 personas adultas, con una prevalencia de miopía de $5.5 \%$ (2004). Además, múltiples estudios de LASIK han reportado en promedio que el $92 \%$ de ojos con miopía o astigmatismo miópico. ${ }^{25}$ Sin embargo, en China en el año 2010 se detectó que la miopía constituyó la ametropía más frecuente, la misma que se incrementó con la edad. También en Singapur la miopía fue el error refractivo más frecuente observado en un estudio realizado en escolares en el año $2010 .^{27}$

En cuanto a la agudeza visual, la mayoría de pacientes en su evolución postquirúrgica sin corrección, muestran valores entre $20 / 20$ - 20/25, una minoría con agudeza visual entre 20/50 - 20/80, estos resultados obtenidos a 1 mes posterior a la cirugía; mostrándose mejores resultados en el lapso de 3, 6 hasta 12 meses posteriores a la cirugía, no se pudo evidenciar dichos resultados ya que el estudio se realizó en un periodo corto y no fue posible realizar un seguimiento en los meses mencionados. La diferencia marcada de los valores prequirúrgicos sin corrección en que la mayoría mantenía una agudeza visual mayor o igual a 20/200. En el estudio de Perdomo F, se observó que la cirugía produjo una mejoría estadísticamente significativa $(\mathrm{p} \leq 0.001)$ en los pacientes, ya que pasaron de promediar una Agudeza visual en el preoperatorio de 0,190 o (20/100) para el ojo derecho y 0,195 o (20/100) para el ojo izquierdo, a promediar una agudeza visual final postoperatoria de 0,941 o $(20 / 20)$ y 0,925 o $(20 / 20)$ respectivamente. Teniendo en cuenta que la mejoría postoperatoria no depende si se trata del ojo derecho o izquierdo, ya que no existe significancia de que la cirugía tenga más éxito en un ojo específico. $^{25}$

Múltiples estudios de LASIK han reportado en promedio que el $92 \%$ de ojos con miopía o astigmatismo miópico, después de la cirugía quedan con defectos menores de 0.5 $\mathrm{D}$, además indican que aproximadamente un $94 \%$ de los ojos operados alcanzan una visión sin corrección de 20/40 o mejor, algunos alcanzan la estabilidad en el transcurso 
de $1,3,6$ o 12 meses posteriores a la cirugía $(26,30,31)$. La recuperación posoperatoria es más rápida en 4 o 6 horas posteriores a la cirugía, en el caso de LASIK; con un tiempo un poco más tardío por la técnica de PRK, en un lapso de 3 a 5 días. Incluso por la técnica de LASIK se mantiene una buena superficie refractiva con un mínimo de distorsión y se alcanza una visión dentro de las 24 o 48 horas después de la cirugía con una agudeza visual de 20/30 o mejor.

La refracción se estabiliza en un término de 6 semanas a 3 meses y existe la posibilidad de volver a realizar la técnica, en el caso de que no se logre una buena agudeza visual. ${ }^{25}$ La eficacia se determina por la agudeza visual sin corrección que presentará posterior a la cirugía refractiva; se informa en pacientes que logran una agudeza visual sin corrección igual o mejor a 6/6 (equivalente a 20/20 en notación de Snellen), que es la visión media estadística de la población, junto con la proporción que logra una agudeza visual sin corrección postoperatoria igual o mejor a 6/12 (equivalente a 20/40 en la notación de Snellen), que es el umbral funcional para la conducción de automóviles y la misma que se ha demostrado que pueden llegar a valores 20/20 o 20/25. Muestran estudios que en PRK se logra una agudeza visual de 6/12 (lo que corresponde a 20/40) o mejor en $85 \%$ de los ojos y en LASIK alrededor de $90 \%$, la misma agudeza visual. ${ }^{28,29}$

Aunque el LASIK reduce el error refractivo y mejora la agudeza visual sin corrección en casi todos los casos; ${ }^{20}$ en el estudio de Kanellopoulos AJ. (2017) a los 3 meses, solo el $86,4 \%$ del grupo LASIK presentaron una visión 20/20 sin corrección. ${ }^{30}$

En el presente estudio no se observaron en el total de pacientes complicaciones transoperatorias. Y la mayoría de pacientes no presentaron complicaciones postoperatorias, ya que las complicaciones se presentan a largo plazo y el seguimiento se debería realizar a 3, 6 o 12 meses posteriores a la intervención, por el periodo corto del estudio no se permitió observar si presentaron complicaciones a largo plazo; una minoría presentaron ojo seco, que es una de las complicaciones más comunes sobre todo cuando se realiza por la técnica de LASIK, y haze corneal que generalmente son transitorias de 1 a 2 años de duración en el estroma subepitelial anterior, que han sido más comunes después de PRK, que de LASIK, donde la formación de opacidades corneales es rara y más bien localizada en los márgenes del flap. En el estudio de Moreno R, indica que estudios de PRK, la incidencia de opacidad o de haze corneal, es clínicamente significativa y se ha reportado entre $0.5 \%$ a $3 \%$ de casos. Generalmente en la fase postoperatoria precoz, los pacientes de PRK, a menudo presentan dolor y disconfort ocular. Otra de las complicaciones es la posibilidad de infección, aunque por las técnicas de PRK y LASIK es rara, con porcentajes que oscilan entre $0.02 \%$ a $1.5 \%(21,54)$. Otra de las complicaciones de ambas técnicas que son poco frecuentes como el síndrome de ojo seco y síntomas visuales nocturnos, como halos y deslumbramiento. Algunos pacientes presentan un error refractivo residual, o regresión del efecto, que constituyen también complicaciones de la cirugía, por lo que pueden requerir un retratamiento. Por lo tanto, el grado de defecto refractivo que justifique un retratamiento dependerá del estilo de vida y expectativas del paciente, se recomienda esperar un período de estabilidad de al menos unos 6 meses post LASIK, tiempo que post PRK puede ser mayor, incluso de hasta 12 meses para observar una estabilidad en la refracción o considerar un retratamiento. ${ }^{20}$

\section{Conclusiones}

La mayoría de los defectos refractivos se encuentra entre una edad de 18 a 25 años de edad, el sexo femenino fue el que mayor tasa de defectos presento.

La ametropía más común fue el astigmatismo miópico, seguida de miopía con una menor frecuencia de casos, la técnica más utilizada es LASIK, se observó una mejoría en la agudeza visual y una disminución en el espesor corneal, no se observaron complicaciones postquirúrgicas.

El alto índice de satisfacción post quirúrgica junto con la baja tasa de complicaciones nos lleva a concluir que cirugía refractiva es una opción terapéutica que provee de resultados positivos, produciendo un cambio psicosocial en las personas y dando una mejor calidad de vida, logrando agudezas visuales de $20 / 20$

\section{Referencias Bibliográficas}

1. García M. Cirugía refractiva sustractiva corneal en la población présbita. Universidad Autónoma de Madrid. Madrid; 2016. Available from: https://goo.gl/YodKvm.

2. Velarde J. Sobre el encurvamiento periférico en la topografía corneal tras cirugía refractiva (LASIK) para corrección de miopía y la relación con parámetros oculares biomecánicos y quirúrgicos. Universidad de Cantabria; 2014. Available from: https://goo.gl/7QZrMr.

3. Miranda I, Barroso R, Perea L, Y R. Selección del paciente para cirugía refractiva: actualización. $\mathrm{Rev} \mathrm{Cu}-$ bana Oftalmol. 2015;28(3). Available from: https://goo. gl/tWQqs6.

4. del Ojo IN. La Miopía. Institutos Nacionales de la Salud; 2013. Available from: https://goo.gl/GMyqhX.

5. Boyd K. ¿Qué es el Astigmatismo? American Academy of Ophthalmology; 2013. Available from: https://goo.gl/ U5Xvz5.

6. Río M. La oftalmología desde la antigüedad. Cuba;. Available from: https://goo.gl/A6eAGy.

7. Tejerina V. Cirugía Refractiva Personalizada: Indicaciones y Resultado del Tratamiento Guiado por Frente de Ondas Topográfico; 2012. Trabajo Fin de Máster en Optometría Avanzada y Ciencias de la Visión. Available from: https: //goo.gl/LxyTdj.

8. Sahbaz I, Tolga M, Kağan G. A Comparative Study between conventional LASIK and IntraLASIK Surgeries based on Sigma Quality Levels; 2016. 
9. Yuksel N, Bilgihan K, Hondur A, Yildiz B. Long term 24. Rey D, Álvarez C, Moreno J. Prevalencia y factores result sof Epi-LASIK and LASEK for myopia. Cont Lens Anterior Eye; 2014.

asociados a miopía en jóvenes. Revista Mexicana de Oftalmología;91(5):223-228.

10. Plata U. Informe de la salud visual y ocular de los países 25. Perdomo F. Evaluación del cambio en la calidad de vida de que conforman la Red Epidemiológica Iberoamericana para la Salud Visual y Ocular. REISVO; 2015.

11. Escalona M, Torres R, Welch G, Martínez J, Zerquera T. Corrección de la miopía mediante cirugía Lasik. Rev Cub 26. Med Mil. 2014;43(1):33-41. Available from: https://goo. gl/QTJdUY.

12. Sánchez D. Cirugía refractiva. vol. Año VII. Paraguay; 2012. Available from: https://goo.gl/suwUha. pacientes miopes operados con cirugía de láser Excimer corneal. España: Universidad de Salamanca; 2015. Tesis doctoral. Available from: https://goo.gl/oG1B4C.

13. Mian S, Trobe J, Libman H. Visual impairment in adults: Refractive disorders and presbyopia; 2017. Available from: https://goo.gl/uojpok.

14. Río M. La oftalmología desde la antigüedad. Cuba; Available from: https://goo.gl/oPzJNT.

15. Arboleda J. Factores que influyen en la aparición de queratocono, en pacientes del área oftalmológica del Hospital Provincial Docente Ambato; 2014. BS thesis. Available from: https://goo.gl/H7F2kJ.

16. Li S, Zhan S, Li S. Laser-assisted subepithelial keratec-29. Merayo J. Conceptos actuales en ojo seco. Del síndrome tomy (LASEK) versus photorefractive keratectomy (PRK) for correction of myopia. Cochrane Data base Syst Rev. $2016 ; 2$.

17. De Luque A. Cirugía refractiva;. Available from: https: 30 . //goo.gl/KBy1Ya.

18. Plata U. Informe de la salud visual y ocular de los países que conforman la Red Epidemiológica Iberoamericana para la Salud Visual y Ocular ( REISVO ), 2009 y 2010; 2015.

19. Sánchez-Di Martino D. Cirugía refractiva. Tendencias en medicina. 2012;Año VII(7):87-94. Available from: https: //goo.g1/U8i3CW.

20. Moreno R, Srur M, Nieme C. Cirugía refractiva: indicaciones, técnicas y resultados. Revista Médica Clínica Las Condes. 2010 Noviembre;21(6):901-910. Available from: https://goo.g1/1UuyU8.

21. Brart D, Shalchi Z, McDonald R. Twenty-year follow-up of a randomized prospective clinical trial ofexcimer laser photorefractive keratectomy. Am J Ophthalmol; 2014.

22. Organización Mundial de la Salud. Ceguera y Discapacidad visual; 2014. Available from: https://goo.gl/g6cMgb.

23. Frick K, Joy S, Wilson D. The Global Burden of Potential Productivity Loss from Uncorrected Presbyopia. Ophthalmology. 2015;122(8):1706-1710.

Recibido: 8 de enero de 2018

Aceptado: 13 de septiembre de 2018 Ocular y Córnea (GESOC; 2008. Available from: https: //goo.gl/V11143.

. Kanellopoulos A. Topography-Guided LASIK Versus Small Incision Lenticule Extraction (SMILE) for Myopia and Myopic Astigmatism: A Randomized, Prospective, Contralateral Eye Study. J Refract Surg. 2017;33(5):306312. Modificaciones de la curvatura posterior corneal después de cirugía refractiva láser. Revista Cubana de Oftalmología. 2011;24(1). Available from: https://goo.gl/EzBe6c. demiológicas de las ametropías en escolares de la Escuela Primaria "Lidia Doce Sánchez". Revista Cubana de Oftalmología. 2013;26(2):571-582. Available from: https://goo.gl/A9LnHW.

Comparación de los valores del espesor corneal central según los equipos Lenstar, Galilei y Pentacam. Revista Cubana de Oftalmología. 2012;25(1). Available from: https://goo.gl/sX7HHW.

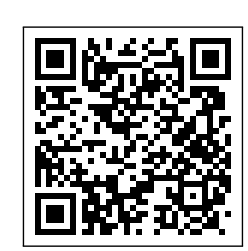

